\title{
Análise do desenvolvimento e atuação do Vórtice Ciclônico de Altos Níveis em janeiro de 2016 no Agreste Meridional, Pernambuco
}

Analysis of the development and performance of the upper tropospheric cyclonic vortices in January 2016 in Agreste, Pernambuco

\author{
BEZERRA ${ }^{1}$, J. F.; SILVA ${ }^{1}$, A. V. R.; ANDRADE ${ }^{1}$, G. S.; $\operatorname{SILVA}^{1}$, N. G.; DUARTE ${ }^{1}$, C. C. \\ josefabiobezerra95@outlook.com
}

\begin{abstract}
Resumo
No presente artigo é exposta uma análise dos eventos anômalos de precipitação pluvial em Janeiro de 2016 no Agreste Meridional de Pernambuco e no Nordeste do Brasil, levando em consideração os mecanismos produtores de tempo que contribuíram para as possíveis anomalias de chuva, tais como Vórtice Ciclônico de Altos Níveis (VCAN), Oscilação Madden-Julian (MJO), Temperatura de Superfície de Mar (TSM) dos Oceanos Pacífico e Atlântico Sul e os impactos desencadeados por esses eventos.
\end{abstract}

Palavras-chave: Vórtice Ciclônico de Altos Níveis; eventos extremos; El Niño

\begin{abstract}
In this article is exposed an analysis of anomalous events of rainfall in January 2016 in Agreste of Pernambuco, taking into account the producers weather time mechanisms that contributed to the possible rainfall anomalies, such as Upper Tropospheric Cyclonic, Madden-Julian Oscillation, Sea Surface Temperature in the Pacific and South Atlantic Oceans and the impacts triggered by these events.
\end{abstract}

Keywords: Upper Tropospheric Cyclonic; extreme events; El Niño.

\section{INTRODUÇÃO}

Os mecanismo e sistemas atmosféricos são dinâmicos e complexos, assim como afirma Sorre (2006, p.91) "Em cada instante dado e em cada ponto do globo, a atmosfera é uma combinação singular que tem muito pouca chance de se reproduzir de uma maneira perfeitamente idêntica. A árvore de meu jardim não florescerá jamais duas vezes nas mesmas condições de temperatura, luminosidade, estado hidrométrico, etc. Não há talvez, no globo, dois locais cujos climas sejam idênticos. [...] (SORRE.p.91, 2006). Em cada lugar da Terra a dinâmica da atmosfera atua de um modo singular, mesmo um sistema de macro escala não modifica o tempo em micro escala de dois ou mais locais de maneira pura, pois as variáveis de micro escala ou conjuntos de mecanismos meteorológicos em cada local moldam o efeito desse sistema.

O estado de Pernambuco foi surpreendido no dia 29 de janeiro de 2016 por uma chuva forte e repentina que provocou danos em alguns municípios, fortes ventos no município de Garanhuns com várias árvores caídas, chuvas de granizo em Arcoverde, no Sertão e em Garanhuns, no Agreste Pernambucano (EUGÊNIO, 2016).

Neste período havia a ocorrência de um El-Ñino intenso, o que intensificou a atuação do chamado Vórtice Ciclônico de Altos níveis (VCAN), o qual, se configura nos altos níveis da 
atmosfera. Segundo Costa (2009), o VCAN é um dos mais importantes sistemas que influencia diretamente o tempo nas regiões tropicais, em especial na região Nordeste do Brasil. Os VCAN são sistemas ambíguos, por produzir chuvas em determinadas localidades (locais sobre a influência da borda do sistema) e ao mesmo tempo inibe a precipitação em outras localidades (locais sobre influência do centro do sistema) (SANTOS, 2015).

A manutenção desse sistema se dá pela conversão de energia potencial em energia cinética através da liberação de calor latente ao longo da periferia, por outro lado, o enfraquecimento do sistema se dá devido à insuficiência de energia cinética (COSTA, 2009). Assim, os VCAN se apresentam como sistemas de intensas convecções, precipitação e nebulosidade nas áreas de periferia (parte quente), enquanto que no centro observa-se subsidência de ar inibindo o desenvolvimento de nuvens (parte fria), ou seja, sua circulação vertical térmica apresenta a ascensão do ar quente nas áreas de bordas e descendência de ar frio nas áreas centrais do sistema.

Em Janeiro de 2016 foi observado um evento de El Niño oscilação Sul intenso e uma atuação de um Vórtice Ciclônico de Altos Níveis (VCAN) de forma expressiva no Agreste Meridional ocasionando diversos transtornos à população da região. De acordo com Harzallah et al. (1986 apud BANDEIRA; MELO, 2016), a frequência de ocorrência dos VCAN tem uma grande dependência da variabilidade interanual da atmosfera e eles são mais frequentes em anos de ocorrência do fenômeno El Niño/Oscilação Sul ENSO. Logo, se pode inferir inicialmente que um dos processos que contribuiu possivelmente na intensidade desse VCAN foi o considerável evento El-Niño associado com as variáveis atmosféricas e com a anomalia da Temperatura da Superfície do Mar do oceano Atlântico Sul que juntos condicionaram a configuração desse sistema.

O presente trabalho tem como objetivo analisar os eventos de precipitações intensas e os sistemas meteorológicos que atuaram no mês de Janeiro de 2016, em especial os Vórtices Ciclônico de Ar Superior, identificando os impactos desencadeados no Agreste Meridional de Pernambuco. Procurar-se-á levar em consideração a atuação de outros possíveis mecanismos que contribuíram para intensificação desses sistemas e para o aumento das chuvas nesse mês, tais como: as anomalias de Temperatura de Superfície de Mar dos oceanos Pacífico e Atlântico e a Oscilação Madden e Julian (MJO).

\section{METODOLOGIA}

Para a identificação e análise da evolução dos sistemas atmosféricos que atuaram no mês de Janeiro foram utilizadas imagens de satélite GOES provenientes do banco de dados do CPTC / INPE (http://satelite.cptec.inpe.br/acervo/goes.formulario.logic). As imagens de satélite selecionadas são dos dias 28 de Janeiro às 23 horas e 30 minutos e 29 de janeiro as 15 horas e 18 
horas no horário relativo ao meridiano de Greenwich (hora universal). O objetivo dessa sequência é comparar e analisar a evolução do Vórtice Ciclônico que avançou no Nordeste entre os dias 28 e 29 desse referido mês.

As informações sobre as alterações na TSM e os dados pluviométricos diários foram obtidos com a Agência Pernambucana de Águas e Climas (APAC) de Pernambuco e informações complementares no site do Climatempo.

Para a análise dos impactos foram coletadas informações nos sites de notícias como o G1 e Blogs da região que apresentaram fotos e relatos dos impactos provocados por estes eventos.

\section{RESULTADOS E DISCUSSÃO}

No Estado de Pernambuco no dia 29 de Janeiro de 2016 ouve o desenvolvimento de um núcleo de instabilidade que influenciou o tempo desde o Litoral até o Sertão. Em algumas localidades, esses eventos foram acompanhados de rajadas de ventos, descargas elétricas, e ocorrência de granizo.

De acordo com jornais e blogs locais houve danos de equipamentos de trânsitos, quedas de árvores e de energia em várias cidades, como nos municípios de Recife (Litoral) e Garanhuns (Agreste Meridional) (EUGÊNIO, 2016).

As chuvas intensas que ocorreram nesse dia foram provocadas pela atuação de um sistema meteorológico denominado de Vórtice Ciclônico de Altos Níveis (APAC, 2016). Devido ao rápido deslocamento desse tipo de sistema, que pode ocorrer em aproximadamente uma hora, não é possível prever esta situação rara com antecedência satisfatória e nem sua intensidade (EUGÊNIO, 2016). No Recife, a velocidade de ventos observada chegou a $80 \mathrm{~km} / \mathrm{h}$, com força suficiente para arrancar uma árvore pela raiz.

O VCAN está associado a nuvens do tipo cumulunimbus, de grande desenvolvimento vertical que podem atingir $12 \mathrm{~km}$ de altura, causando precipitações com intensidade moderada a forte, acompanhadas de trovoadas, descargas elétricas, rajadas de vento e, com menor frequência, a ocorrência de granizo, como ocorreu no Agreste Meridional. Os volumes mais significativos de chuva ocorreram entre $12 \mathrm{~h} 30$ e $15 \mathrm{~h}$ do dia 29/01 (APAC, 2016).

As figuras 1 e 2 referem-se a dois exemplos de danos provocados pelos fortes ventos e pela chuva intensa que ocorreu em 29/01/2016 no município de Garanhuns. 

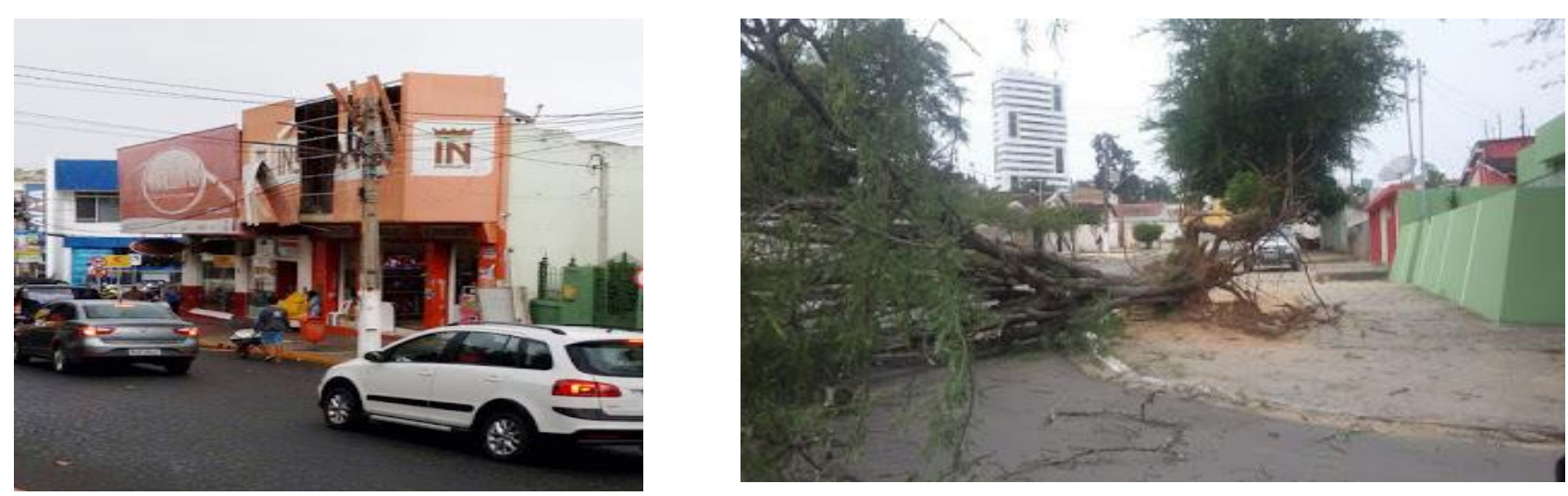

Figura 01. Exemplos de danos provocados pelas fortes chuvas do dia 29/01/2016 no município de Garanhuns, Pernambuco. (A) Fachada danificada pela força do vento da loja Insinuante no município de Garanhuns PE; (B) Árvore tombada pela força do vento no município de Garanhuns. Fonte: Eugênio (2016)

O mês de Janeiro de 2016 foi marcado por um evento de El-Niño forte, que de acordo com a Agência Pernambucana de Águas e Climas (APAC, 2016) o oceano Pacífico Equatorial permaneceu ainda muito aquecido entre a segunda quinzena de dezembro de 2015 e a primeira quinzena de Janeiro 2016, apresentando anomalia positiva da TSM de até $3^{\circ} \mathrm{C}$. Vale salientar que o El-Niño costuma reduzir as chuvas sobre o setor Norte e a região semiárida do Nordeste, no entanto, nesses períodos os Vórtices Ciclônicos de Altos Níveis localizados sobre o Nordeste ou sobre o Atlântico são mais extensos na vertical, atingindo níveis de $500 \mathrm{hPa}$, enquanto que em anos de La Niña esses sistemas se encontram confinados ou restritos em altos níveis (SANTOS, 2015).

Em relação a temperatura da superfície do mar (TSM) da bacia do Atlântico Sul de acordo com as informações coletadas no site da Apac (APAC, 2016) observou-se um aquecimento em uma faixa de água que se estende desde a costa da África até o litoral norte da Região Nordeste, o que favorece o transporte de umidade para essa área. Além disso, nesse período, a oscilação MaddenJulian influenciou a região Nordeste e contribuiu para o aumentos das chuvas. Essa oscilação é definida como um deslocamento de oeste para leste de uma célula zonal de grande escala, que causa alterações na convecção tropical (MELO, 2016).

De acordo com o Climatempo (2016) no mês de Janeiro ouve o deslocamento de uma onda intensa avançando desde o Pacífico Oeste em direção ao Continente Africano, tendo sua fase denominada fase 8, favorecendo o aumento das chuvas sobre o Nordeste Brasileiro (NEB). Assim, infere-se que a intensificação dos sistemas que atuaram sobre o NEB em Janeiro de 2016 também tiveram influência dessa oscilação.

A figura 2 (a, b, c e d) apresenta a evolução do VCAN nos dias 28 e 29 de janeiro de 2016, sobre o Nordeste do Brasil, mais precisamente sobre o Estado de Pernambuco, do Litoral ao Agreste. Observa-se uma mudança repentina na configuração do VCAN sobre o NEB (Figura 2c e 
2d), em que há um desenvolvimento máximo (Figura 2d) do núcleo convectivo de nuvens cumulunimbus nas áreas próximas ao centro do sistema avançando sobre o estado de Pernambuco desencadeando os eventos de temporais sobre o Litoral e Agreste Pernambucano.

O VCAN é fácil de ser identificado nas imagens de satélite devido ao seu movimento horário que as nuvens convectivas em sua periferia realizam devido às configurações dos ventos a 10 km de altitude, porém, neste dia 29 às 18h GMT (Figura 2d), às 15 horas em Pernambuco, há uma evolução interessante nas áreas centrais desse sistema ao avançar sobre o continente. O que faz disso um evento interessante, é que nas áreas centrais desse sistema devido à subsidência de ar, há uma redução das instabilidades, no entanto, no dia 29 houve um desenvolvimento de instabilidade próximo ao centro do VCAN devido a um dinamismo atmosférico que produziu uma evolução rápida e inesperada, diferente do esperado, que seria uma redução das chuvas nos próximos dias sobre o NEB (PEGORIM, 2016).

Costa (2009) deduz que um aumento na intensidade da convecção, possivelmente acontecendo próximo ao centro do vórtice, provocará a formação de um núcleo quente que culminaria com a decadência da baixa fria. Considerando-se, assim, a ideia exposta por esse autor pode-se deduzir que embora houvesse descendência de ar no centro do sistema no dia 28 (Figura 2a), em que se observa pouca nebulosidade, a atuação de um possível evento de médio ou micro escala em função das alterações da TSM ou da temperatura sobre o continente, provocaram alterações significativas no dia 29 que desencadeou um núcleo convectivo nas áreas centrais do VCAN (Figura 2c e 2d), alterando a característica de um centro frio em que o ar descendia (baixa fria) para um núcleo quente em que há convecção do ar que elevou a umidade para os níveis mais alto da atmosfera permitindo o desenvolvimento desse núcleo de instabilidade sobre o Nordeste.

Assim, analisando a Figura 2a em que apresenta a imagem do dia 28 de janeiro às $23 \mathrm{~h} 30$ GMT (20 horas e 30 minutos no horário local) mostrando a entrada do VCAN sobre o Nordeste; a Figura 2b refere-se ao dia 29 de janeiro às 12h30 GMT (9 horas e 30 minutos no horário local), mostrando o centro do sistema sobre parte do NEB. Ainda há pouca nebulosidade nas áreas centrais, observa-se a curva feita pela nebulosidade nas áreas periféricas do sistema devido ao movimento horário, caracterizando a presença de um vórtice. A Figura $2 \mathrm{c}$ representa a evolução do sistema no dia 29 às 15 h00 (12 horas referente a hora local), em que se pode observar a formação de um pequeno núcleo nas proximidades do centro do VCAN, precisamente sobre o litoral norte do Estado de Alagoas evidenciando um repentino desenvolvimento de um núcleo convectivo. E por fim, a figura 2d que representa a evolução do sistema no dia 29 às 18 h00 (15 horas em Pernambuco), observa-se o máximo desenvolvimento do núcleo convectivo nas áreas próximas ao 
centro do sistema avançando sobre o Estado de Pernambuco desencadeando os eventos de temporais sobre Litoral e Agreste pernambucano.

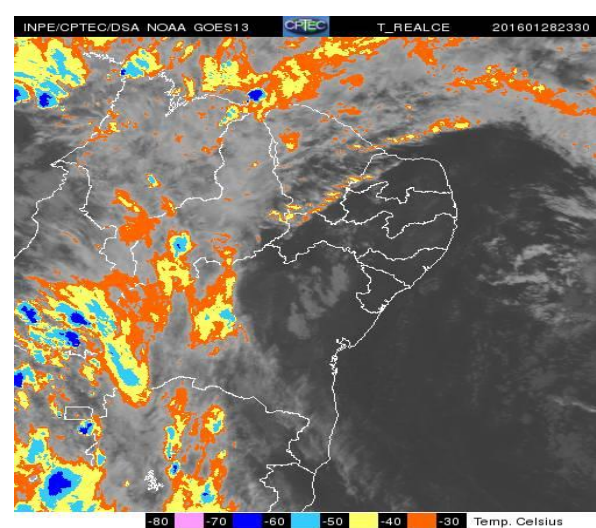

(A)

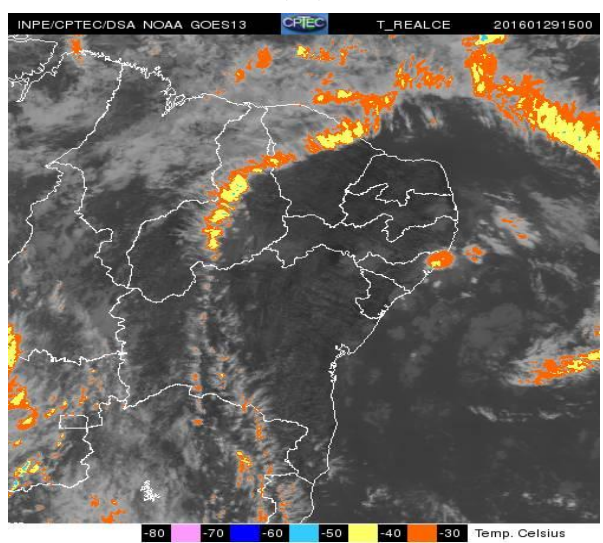

(C)

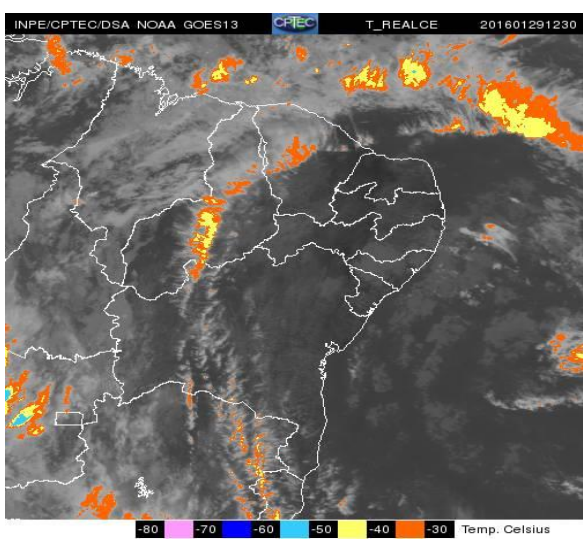

(B)

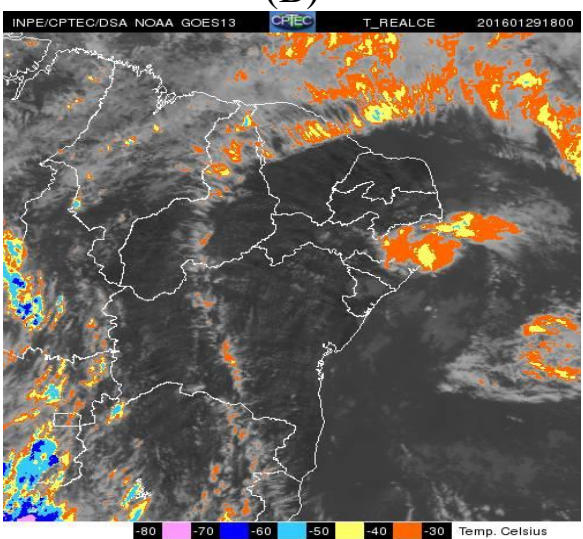

(D)

Figura 02. Evolução do Vórtice Ciclônico de Altos Níveis sobre o Nordeste Brasileiro nos dias 28 e 29 de janeiro de 2016. (a) Imagem de satélite do dia 28 as $23 \mathrm{~h} 30$ GMT mostrando a entrada do VCAN encima do Nordeste; (b) Imagem de satélite do dia 29, 12h30; (c) Imagem de satélite do dia 29 as 15 h00 GMT; (d) Imagem de satélite do dia 29 às 18 h00 GMT. Fonte: CPTEC / INPE

Para concluir a análise também foram observados volumes de chuvas consideráveis na maior parte da Região Nordeste, em praticamente todos os Estados, principalmente na Bahia, áreas de Pernambuco, Alagoas, Ceará, sul do Piauí e sul do Maranhão. A maior parte destas chuvas foi gerada pelos Vórtices Ciclônicos de Altos Níveis associado com as variáveis atmosféricas que influenciam normalmente o tempo na maior parte do Nordeste incluindo Pernambuco e consequentemente o Agreste Meridional. No entanto, em especial no estado da Bahia parte dos elevados índices pluviométricos que se concentraram no início do mês de Janeiro de 2016 deve-se também a entrada de uma frente fria associada com um ligeiro aquecimento do oceano que por volta dos dias 3, 4 e 5, principalmente no dia 4, produziu intensos núcleos de instabilidades que levaram chuvas intensas para a parte Norte e Oeste desse estado e em parte de Sergipe a instabilidade desse sistema frontal influenciou o tempo na tarde do dia 4. 
Tal fenômeno exemplifica a participação de outras variáveis atmosférica que junto com os VCAN que contribuíram para tornar os eventos de Janeiro de 2016 excepcionais em algumas áreas do Nordeste.

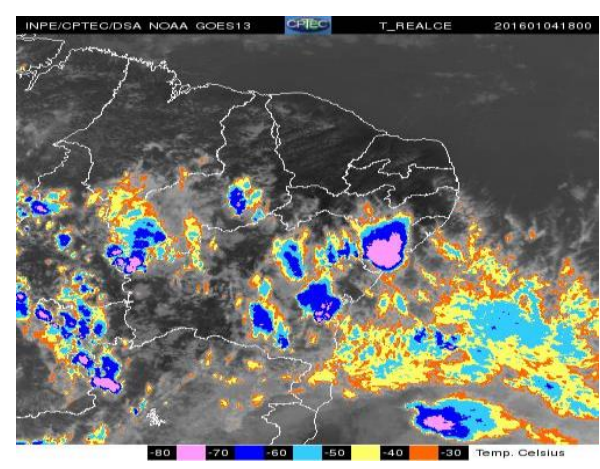

(A)



(B)

Figura 03. Imagens de satélite do dia 4 de Janeiro de 2016 às 18 h00 (A) e às 20h30 (B), demonstrando o desenvolvimento de intensos núcleos de instabilidades (cores azul a rosa) que produziram chuvas intensas. Fonte: CPTEC / INPE

\section{CONSIDERAÇÕES FINAIS}

Ao se estudar a atuação dos diversos sistemas produtores de tempo no Nordeste do Brasil, no caso específico de janeiro de 2016, pode-se concluir que as ocorrências de eventos pluviométricos em um determinado período podem estar associado à atuação de mais de um sistema produtor de tempo, ou estar relacionado a mais de uma variável atmosférica e oceânica atuando em conjunto configurando, assim, tais eventos.

Diante desses fatos, percebe-se que a atmosfera é dinâmica e por essa razão não é possível prever com precisão a intensidade e evolução de um sistema meteorológico, bem como seus possíveis impactos. Sistemas como o VCAN aqui abordado podem ter sido intensificado por outros processos ou sistemas que atuaram em conjunto, tais sistemas podem ser de macro, meso, ou micro escala, ou como no caso específico também influenciados pelas alterações na TSM dos oceanos Pacífico e Atlântico Tropical e pela oscilação Madden e Julian que afetam a pluviometria da região tropical. Esses são detalhes que devem ser levados em consideração e que precisam ser melhor estudados para subsidiar um planejamento urbano e territorial que ajude a população a conviver com a atuação anômala de tais fenômenos. 


\section{REFERÊNCIAS}

APAC. Análise das Condições Oceânicas: Janeiro de 2016. 20 jan. 2016. Disponível em: http://www.apac.pe.gov.br/noticias.php?noticia_id=758. Acesso em: 29/01/2016.

BANDEIRA.M.M; MELO.E.C.S. Atuação do vórtice ciclônico de altos níveis sobre o nordeste do brasil no mês de janeiro nos anos de 2004 e 2006. Anais do Congresso Brasileiro de Brasileiro de Meteorologia. Disponível em: http://www.cbmet.com/cbm-files/145b4fcbf36e03369668796488dcbfd798.pdf. Acesso em: 02/02/2016.

CLIMATEMPO. Influências da oscilação Madden - Julian no verão 2016 do Brasil. 27 jun. 2016. Disponível em: http://www.climatempo.com.br/videos/video/UXrk1_TNWcg. Acesso em: 19 jun. 2016.

COSTA, B.S. Balanço de vorticidade e energia aplicado aos Vórtices Ciclônicos de Altos Níveis atuantes no Oceano Atlântico Tropical Sul e adjacência. Dissertação de Mestrado apresentada ao Instituto de Astronomia, Geofísica e Ciências Atmosféricas/Universidade de São Paulo, 2009, 100 p.

EUGÊNIO, Carlos. GARANHUNS: Chuva e rajadas de vento derrubam árvores e danificam fachadas de lojas. Queda de granizo também é registrada. Garanhuns, 29 jun. 2016. Disponível em: blog:http://www.blogdocarloseugenio.com.br/2016/01/garanhun s-chuva-e-rajadas-de-vento.html. Acesso em: 19 jul. 2016.

PEGORIM, J. VCAN e ASAS atuam sobre o Brasil. 29 jul. 2016. Disponível em: http://www.climatempo.com.br/noticia/2016/01/28/vcan-e-asas-atuam-sobre-o-brasil-5218. Acesso em: 29 jul. 2016.

MELO. L.M: A Oscilação Madden e Julian (MJO) e Sua Influência Sobre o Centro-Oeste do Brasil. Anais do Congresso Brasileiro de Brasileiro de Meteorologia. Disponível em: http://www.cbmet.com/cbm-files/14-e4fc3ec4930e27b49b73d97d25848da8.pdf. Acesso em: 02 fev. 2016.

SORRE.M. Objeto e Método da Climatologia. Revista do departamento de Geografia. 2006, n. 18. São Paulo: 2006, p. 89-94. Traduzido por: Prof. Dr. José Bueno Conti. 
SANTOS. A.L: Acurácia do modelo regional ETA utilizado no CPTC/INPE em detectar os Vórtices Ciclônicos de Altos Níveis atuantes no Nordeste do Brasil. Dissertação de Mestrado apresentada ao Programa de Pós-Graduação em Meteorologia / Instituto Nacional de Pesquisas Espaciais, 2015, 101 p.

\section{AGRADECIMENTOS}

Os autores agradecem ao Centro de Previsão de Tempo e Estudos Climáticos (CPTEC) do Instituto Nacional de Pesquisas Espaciais (INPE) pelo fornecimento das imagens de satélite. A Agência Pernambucana de Água e Climas (APAC), ao Climatempo e ao Instituto Nacional de Meteorologia (INMET). Ao Departamento de Geografia da Universidade de Pernambuco - Campus Garanhuns pelo incentivo a pesquisa.

Recebido em: 14/08/2016

Aceito para publicação em: 01/10/2016 